\title{
Recorrido y actualización necesaria por la perspectiva cualitativa
}

Routeway and necessary update on the qualitative perspective

Maria Agustina Coloma

DOI: https://doi.org/10.24215/18537863e074

Instituto de Investigaciones en Humanidades y Ciencias

Sociales (Universidad Nacional de La Plata/CONICET),

Argentina

colomaagustina@gmail.com

Verd J. M, Lozares C. Introducción a la investigación cualitativa: fases, métodos y técnicas. 2016. Madrid. Síntesis. 372pp.

\section{Resumen:}

En esta reseña sobre el libro Introducción a la Investigación cualitativa: Fases, métodos y técnicas, de los autores Verd y Lozares, intentamos presentar los temas que aborda la obra y también compartir una apreciación respecto a sus principales contribuciones y aportes. Entendemos que la obra cobra relevancia por la manera en que esboza el desarrollo del proceso investigativo, desde las preguntas iniciales hasta la confección de los informes. Renueva algunas respuestas a interrogantes clásicos de la metodología, se posiciona sobre los estándares de calidad necesarios y la importancia de la toma de decisiones autónomas y reflexivas para la producción científica.

Palabras clave: Metodología cualitativa, Técnicas de investigación, Lógica abductiva.

\section{Abstract:}

In this review of the book Introducción a la Investigación cualitativa: Fases, métodos y técnicas, by the authors Verd and Lozares, we try to present the issues addressed in this work and also share an appreciation regarding their main inputs and contributions. We understand that the book becomes relevant for the way it outlines the development of the research process, from the initial questions to the preparation of the reports. It renews some answers to classic questions from the methodology field, it is positioned on the quality standards required and the importance of making autonomous and reflective decisions for scientific production.

KEYWORDS: Qualitative methodology, Research techniques, Abductive logic.

\section{INTRODUCCIÓN}

En esta obra se presentan de manera completa y cuidadosa las perspectivas y técnicas utilizadas en la investigación cualitativa. Introducción a la investigación cualitativa... se vuelve una actualización necesaria y lectura recomendada para quienes estén interesados en aprender sobre estas temáticas, como así también para quienes ya conozcan del tema y quieran realizar un ejercicio reflexivo y una meditación disciplinar y actualizada.

En la obra, los autores van presentando los elementos, explicándolos en detalle y relacionando todos los mecanismos hasta llegar a presentar un panorama muy completo de los aspectos que dan sentido a la investigación cualitativa. Sin evitar temas controvertidos, presenta variedad de perspectivas y sugiere numerosas fuentes en caso de querer ampliar en algún tema específico.

Además, el texto nos invita a consultar otras secciones dentro de él, cuenta con numerosas referencias cruzadas que nos permiten navegar en el texto retrocediendo o avanzando cuando hemos consultado la obra en función de una temática o capítulo específico.

Al mismo tiempo, se presentan actualizaciones bibliográficas sobre los cánones de la metodología, actualizaciones temáticas incorporando perspectivas y problemáticas contemporáneas, como el uso de los recursos virtuales e internet, o la reflexión sobre el uso de paquetes informáticos para el análisis de la información. 


\section{Características del TeXto}

Introducción a la Investigación Cualitativa. Fases métodos y técnicas cuenta con un prólogo de Miguel Valles que señala una línea de continuidad entre el afamado manual del prologuista, publicado en 1997 (Valles, 1997), que se ha cristalizado como una literatura de referencia básica de los aprendices de ciencias sociales, y esta obra que se edita 20 años después de aquel éxito.

En las palabras introductorias a cargo de los autores, el escrito se presenta como una herramienta para “aprender a tomar decisiones" (p. 18) y, a su vez, como un texto destinado fundamentalmente a estudiantes. Está dirigido tanto a aquellos alumnos que se encuentran en primeros años de la universidad y ven estos temas por primera vez, como a quienes deben realizar tesinas o tesis de máster y también a estudiantes de doctorado.

El libro se encuentra organizado en trece capítulos distribuidos en cuatro partes. La primera funciona como una presentación general a los métodos cualitativos y al mismo tiempo plantea las claves de lectura para el resto del libro. Las tres siguientes corresponden a las diferentes fases de la investigación: el diseño, la recolección y el análisis.

Para ver con un poco más de detalle cada apartado podemos empezar comentando los capítulos que conforman la primera parte.

El primer capítulo, titulado "La investigación cualitativa el qué y el para qué”, es el que posee más desarrollo de la epistemología y dialoga con los principios filosóficos de la perspectiva, pero a la vez logra generar puentes y distancias con la perspectiva cuantitativa. Asimismo, identifica las tradiciones teórico-metodológicas que han aportado en el desarrollo de la investigación cualitativa y presenta las características que componen a esta tradición. Se exhibe una serie de situaciones en las que es preciso utilizar las herramientas de la investigación cualitativa.

En el siguiente capítulo, "Las estrategias metodológicas en la investigación cualitativa",los autores profundizan sobre las maniobras posibles a la hora de llevar adelante investigaciones de este estilo, pero, más importante aún, nos presentan tres formas lógicas que dialogan con las estrategias, técnicas y métodos. Estas formas son la lógica deductiva, la lógica abductiva y la lógica inductiva. A partir de estos esquemas de organización clasificaremos una investigación según el lugar que la teoría tiene en ella, de esta manera organizaremos el resto del sendero; los autores plantean que las decisiones tomadas respecto al lugar de la teoría determinan la estructura de la investigación.

En el caso de la lógica deductiva la teoría se presentará antes de la empiria, y esta variante que está muy asociada a los métodos cuantitativos también tiene su versión cualitativa. De otra forma, una investigación puede estructurarse de manera opuesta, dando prioridad temporal a la empiria sobre la teoría; en este caso la teoría será producida a partir de generalizaciones empíricas y la lógica que prima es la inductiva.

El diseño a partir de la lógica abductiva postula un punto de encuentro intermedio en el cual teoría y empiria se articulan y dialogan para dar lugar a generalizaciones empíricas y teorizaciones. Estos conceptos serán una clave de lectura muy interesante para el resto de la obra.

El capítulo final de la primera parte se titula "El proceso de la investigación cualitativa y sus fases".En él se anuncian las etapas de toda investigación (preparatoria, trabajo de campo, analítica, informativa) y las distintas maneras de abordarlas según diferentes esquemas de diseño de investigaciones (cerrado, flexible o emergente). A continuación, se profundizará en las herramientas propias de cada etapa haciendo visibles las lógicas detrás de cada paso y decisión elegida.

Verd y Lozares llevan la atención, en este apartado, hacia la coherencia interna de las investigaciones, al tiempo que reflexionan sobre los pasos necesarios a seguir de acuerdo a cada uno de los posibles diseños y a los procesos lógicos para consumar una investigación científica. Además, realizan una interesante contribución al sintetizar los procesos y objetivos de cada una de las fases y exponer los productos a los que se debe apuntar como resultado de cada una de ellas. 
La segunda parte del libro se ocupa especialmente del diseño de la investigación y está compuesta por dos capítulos.

"La pregunta de investigación y la preparación del estudio" es el primero. El tratamiento que se da al paso del tema al problema aborda un tópico que será de gran utilidad para toda persona que está empezando a realizar ese ejercicio, ya que enseña la potencia de la confección de interrogantes y las posibilidades del diálogo con diferentes perspectivas teórico-metodológicas.

En este capítulo también se brindan recomendaciones sobre el uso de fuentes bibliográficas en la etapa del diseño. Tal como fue dicho previamente por los autores, el uso de las fuentes literarias en la construcción del objeto dependerá tanto del tipo de diseño como de la forma lógica utilizada, pero sin duda hay interrogantes -más o menos abiertos- que se hace indispensable responder en cualquier tipo de diseño cualquiera sea su grado de estructuración.

El siguiente capítulo de esta sección, "La selección y el acceso a las unidades de información", versa sobre lo que comúnmente llamaríamos muestreo, y se autodefine como la bisagra entre la etapa de diseño y la de campo, a partir de aquí empieza a profundizarse aún más en las características de la investigación cualitativa, retoma aportes de la teoría fundada ${ }^{1}$ y presenta las distintas variantes para la selección de los casos destacándose los aportes de la Escuela Cualitativista de Madrid.

Seguidamente nos encontramos con la audacia de los autores para ensayar una respuesta a la siempre presente pregunta de cuántas unidades debe tener un estudio cualitativo. Al finalizar esta sección se abordará el acceso al campo y las cuestiones éticas de la confidencialidad y el consentimiento.

La tercera parte de la obra se titula "Los métodos de obtención de datos", y dedica los cinco capítulos siguientes a caracterizar las diferentes técnicas de recolección. La maniobra para cada una de ellas es realizar una presentación de la historia y los antecedentes de cada técnica, delinear sus características, atender a las particularidades de cada una y concluir con una síntesis de ventajas y desventajas de las mismas.

Los métodos desarrollados a lo largo de esta sección son la entrevista, el método narrativo-biográfico, el de discusión en grupo, la observación cualitativa y el uso de documentos, dedicando un capítulo a cada uno. Que los autores hayan decidido tomar un capítulo para al método narrativo biográfico y no sumarlo como una variante de la entrevista nos habla del diálogo con la realidad disciplinar y actualidad de la obra, como también lo hace la incorporación de respuestas sobre los desafíos de las entrevistas por medios virtuales, la etnografía virtual y otros registros digitales útiles en investigación cualitativa.

Cada uno de los capítulos específicos explicita el cómo, el por qué y el para qué de las técnicas de manera que los lectores puedan encontrarse bien orientados para generar los vínculos entre los objetivos, la producción de datos y el análisis. En esta sección podemos encontrar plasmada, de manera muy interesante, la trasmisión de saberes actitudinales que muchas veces se trasfieren de manera oral e informal a quienes se inician en investigación cualitativa. Este texto recoge esa inquietud y elabora una posible solución para su transmisión, una muestra, seguramente, de la orientación pedagógica de esta obra.

La cuarta parte alberga los dos capítulos finales de la obra, uno de ellos dedicado al análisis de información y el otro a la presentación de resultados y el informe final.

Lejos de plasmar fórmulas extraordinarias sobre cómo debe analizarse la información, se ofrecen ejercicios y se proponen tareas orientadas hacia la especificidad de lo cualitativo; los mismos se orientan hacia un ejercicio reflexivo que ponga en conexión objetivos, información, y que se encuentre abierto a reflexionar de cara a los puntos de vista de aquellos a los que se ha acudido para producir la información. En este capítulo se abordan las diferentes técnicas de análisis en conexión con las escuelas en las que han surgido, sus grados de autonomía y las orientaciones más importantes para el análisis. Es aquí donde nos encontramos con un apartado dedicado a la actualidad de los CAQDAS, los paquetes informáticos dedicados a la asistencia en el análisis cualitativo.

Para la redacción de tesis o informes de investigación la aproximación brindada en "La presentación de los resultados y la redacción del informe final” se articula en torno a los cánones de calidad y operatividad, la 
necesidad de comunicar resultados y argumentación que sirvan para realizar intercambios y entrar en diálogo con la audiencia específica para la que se produce el texto.

\section{Cierre}

En esta obra se busca dotar al lector de los conocimientos y las referencias para poder desarrollarse con autonomía en el terreno de la investigación. Los autores ofrecen un completo abanico de los elementos presentes en el desarrollo de la investigación cualitativa, y hacen el esfuerzo de conectarlo con las tradiciones más importantes del s. XX.

Resulta de utilidad pensar por qué seguimos necesitando de obras de estas características. En el caso de esta obra nos da pie a preguntarnos sobre la calidad de las producciones y reflexionar sobre los estándares de validación, la reflexividad propia de la tarea y la conciencia de las decisiones adoptadas.

\section{Bibliografía}

Valles, M. S. (1997). Técnicas cualitativas de investigación social. Reflexión metodológica y práctica profesional. Madrid: Síntesis.

\section{Notas}

1 Se conoce como Teoría Fundada, Teoría Fundamentada (o en inglés Grounded Theory) a una serie de aportes realizados en los años 60, por sociólogos de la Escuela de Chicago. Los principales referentes de esta corriente son Glaser y Strauss y sus aportaciones versan en torno al diseño de las investigaciones, el muestreo cualitativo y la importancia de la labor interpretativa del científico social. Para la teoría fundada la obtención de datos y la labor interpretativa están íntimamente conectadas; se opusieron a la visión positivista más extrema que no cuestionaba el carácter subjetivo de la percepción de la realidad y tampoco daba importancia a las interpretaciones propias de los sujetos estudiados.

\section{BY-NC-SA}

